\title{
Visual Models for Abstract Concepts towards Better Learning Outcomes and Self-Efficacy
}

\section{Dr. K. Jo Min, Iowa State University}

K. Jo Min is an Associate Professor and Director of Undergraduate Education in the Department of Industrial and Manufacturing Systems Engineering at Iowa State University. He teaches courses in sustainable production systems and market-based allocation mechanisms. His education research interests include continuous improvement for objectives and outcomes, teaching and learning of global enterprise perspectives, and international student team management and effectiveness. His research publications have appeared in International Journal of Engineering Education, The Engineering Economist, IEEE Transactions on Engineering Management, and others.

\section{Dr. John Jackman, Iowa State University}

John Jackman is an Associate Professor of Industrial and Manufacturing Systems Engineering at Iowa State University. His research interests include engineering problem-solving, computer simulation, webbased immersive learning environments, and data acquisition and control.

\section{Jason C.K. Chan}




\title{
Visual Models for Abstract Concepts towards Better Learning Outcomes and Self-Efficacy
}

\begin{abstract}
We constructed and analyzed an evidence-based practice case to see if visual models help students develop a better understanding of abstract concepts and enhance their self-efficacy when solving engineering problems. Abstract concepts without corresponding physical phenomena are often found in the domains of industrial engineering, engineering management, and systems engineering. In this study, we focus on inventory control of a supply chain, which is typically a junior level undergraduate production systems course in an industrial engineering program. Visual models of inventory behaviors were designed to complement the traditional approach of mathematical derivations and numerical computations. In this context, we use a randomized-controlled design research framework implementing the visual models in a quiz. Pre- and post-surveys on student self-efficacy were used to assess the effects of the visual models. Students' quiz outcomes and self-efficacy surveys are compared to those from a control group that did not use the visual models, and the results from both groups were statistically analyzed. This study is motivated by engineering students' inability to understand abstract concepts and the need for continuous improvement of student learning. The results show that, within the scope of the aforementioned experiment and collected data, the visual models do help students understand abstract concepts and improve their self-efficacy. This study can serve as a basis for further studies on the extent of visual models helping students develop a complete mental model and on whether better mental models actually lead to a better understanding of the domain knowledge and enhance students' self-efficacy.
\end{abstract}

Keywords: Abstract Concepts, Visual Models, Learning Outcomes, Self-Efficacy

\section{Introduction and Objectives}

\begin{abstract}
concepts without direct physical representations related to principles of engineering economics and management are difficult for engineering students to conceptualize as evidenced by their inability to explain their solutions. We observe that efforts to improve the learning outcomes of such students have included a substantial increase in the use of visual models for abstract concepts in textbooks, DVDs, and online resources.
\end{abstract}


To our knowledge, however, there has been little systematic research on whether and how visual models help engineering students better understand abstract concepts especially in the areas of industrial engineering, engineering management, and systems engineering. To address this issue from an engineering education research perspective, two essential questions are (1) to what extent do visual models of such concepts help students develop a complete mental model and (2) whether better mental models lead to better understanding of the domain knowledge and enhance students' self-efficacy.

Towards answering these important questions, we explore the effects of visual models on students' understanding of domain knowledge and their self-efficacy in the specific context of inventory control theory. This study is motivated by our preliminary conjecture that students' mental models might be enhanced when visualization complements mathematical formulations and solutions. This is consistent with Hong and O'Neil ${ }^{1}$ who found that simple diagrams helped students develop mental models of statistical confidence intervals. The importance of visualization in student learning can also be seen in the large increase in graphs and diagrams in teaching materials in recent years (e.g., Wheat ${ }^{2}$ reports that a macroeconomic textbook containing 200-400 graphs is not uncommon). When visualization is needed for abstract concepts with few intuitive physical representations, however, we have observed that there are few, if any, graphs and diagrams.

The rest of the paper is organized as follows. In the next section, we briefly review the existing literature relevant to this study. This is followed by a description of our research scheme in the context of inventory control theory and the relevant test contents. We next explain the assessment of the test results, followed by the pre- and post- surveys for students' self-efficacy and their corresponding assessment results. We then provide concluding remarks and comment on future research.

\section{Literature Review}

There have been numerous studies related to visual learning styles and the benefits of visualization (see e.g., Felder ${ }^{3}$ ). Tall ${ }^{4}$ found that, when students drew graphs that represented physical representations (e.g., slope or area), they developed a better understanding of calculus. There are many examples of visualization tools that were developed to aid student 
learning in engineering education. For example, Heath et al. ${ }^{5}$ suggested that the visual display of performance data on parallel computing would be important for student comprehension. Wood $^{6}$ developed software for visualizing concepts related to digital logic design and digital signal processing. The goal was to help students understand basic concepts in the context of electrical engineering. Assessments of improvement in student learning were not provided. Extensive research has demonstrated the efficacy of visual aids on students learning across a variety of domains, including learning verbal materials, spatial layout, sports rules, mechanical structures, etc. (e.g., Novick et al. ${ }^{7}$ ).

One reason why visual models improve student understanding is that visual cues help learners offload part of the conceptual processing required to the visuospatial domain, thus freeing up valuable verbal resources in working memory (Haugwitz et al. ${ }^{8}$ ). Modern theoretical models of working memory typically consists of three components, a central executive responsible for attention deployment, a phonological loop responsible for temporarily holding verbal information in short-term memory, and a visuospatial sketchpad responsible for storing visual information (e.g., colors, shapes) and spatial relations among objects (Baddeley ${ }^{9}$ ). Working memory capacity is predictive of mathematics performance and general fluid intelligence (Bull and Scerif ${ }^{10}$ ). Visualization may help reduce overloading of the phonological component of working memory, which is crucial to performing complex mathematical operations (e.g., holding intermediate values in mind while performing other important calculations). Another reason that visual representation enhances problem solving is by turning abstract concepts into concrete spatial layouts (Winn ${ }^{11}$ ) and by exchanging inefficient sentential representations (which are sequential and thus slow) for easier perceptual representations (Larkin and Simon ${ }^{12}$ ).

As for mental models, several theories have been proposed to explain how visualization improves mental modeling from a cognitive perspective. Crapo et al. ${ }^{13}$ theorized that students try to reconcile their mental models with the visualization and make changes in their mental models based on any disparities. The challenge for an empirical study is that mental models are not directly observable. Therefore, students need to externalize their mental models in order to collect data and analyze the models. During problem solving, students try to understand a scenario by constructing representations (i.e., mental models) that help them understand what is happening in the scenario. 
Due to its effectiveness in promoting learning, mathematical educators have advocated increased use of visual aids in the classroom (Barwise and Etchemendy ${ }^{14}$ ), but visualization aids for abstract concepts in engineering have not been as widely adopted. In industrial engineering, systems engineering, and engineering management domains (which all share common interests in supply chains), the impact of visualization on learning abstract concepts has not been studied. Given that self-efficacy has been closely correlated to cognitive engagement and performance (see e.g., Pintrich and de $\operatorname{Groot}^{15}$ ), the relationship between visual models and enhanced self-efficacy needs to be further investigated.

\section{Methodology}

We conducted a randomized study as follows. A problem solving session for inventory control theory was designed for junior level undergraduate industrial engineering majors. We also conducted pre- and post- self-efficacy surveys on students' abilities regarding the specific domain knowledge aspects of inventory control theory.

\section{Participants}

Students in the class were divided randomly into 2 groups, A and B. In Group A, 44 students completed the problems and in Group B, 42 students completed the problems. Both groups had originally been designed for 45 students each, but last-minute sickness, etc., led to less than $100 \%$ completion. For Group A, the problems on the inventory control theory were accompanied by relevant visual models. For Group B, the same problems were given without the figures.

\section{Procedure}

The problems were given to each group at the same time, but in different classrooms. There were two separate problems that participants were asked to solve. In the first problem (No. 1b), both Group A and Group B students were asked if the optimal reorder point derived from No. 1a through an iterative, computational algorithm could be greater than the corresponding order quantity, and explain the reason. Group A was given the visual model in Figure 1. 


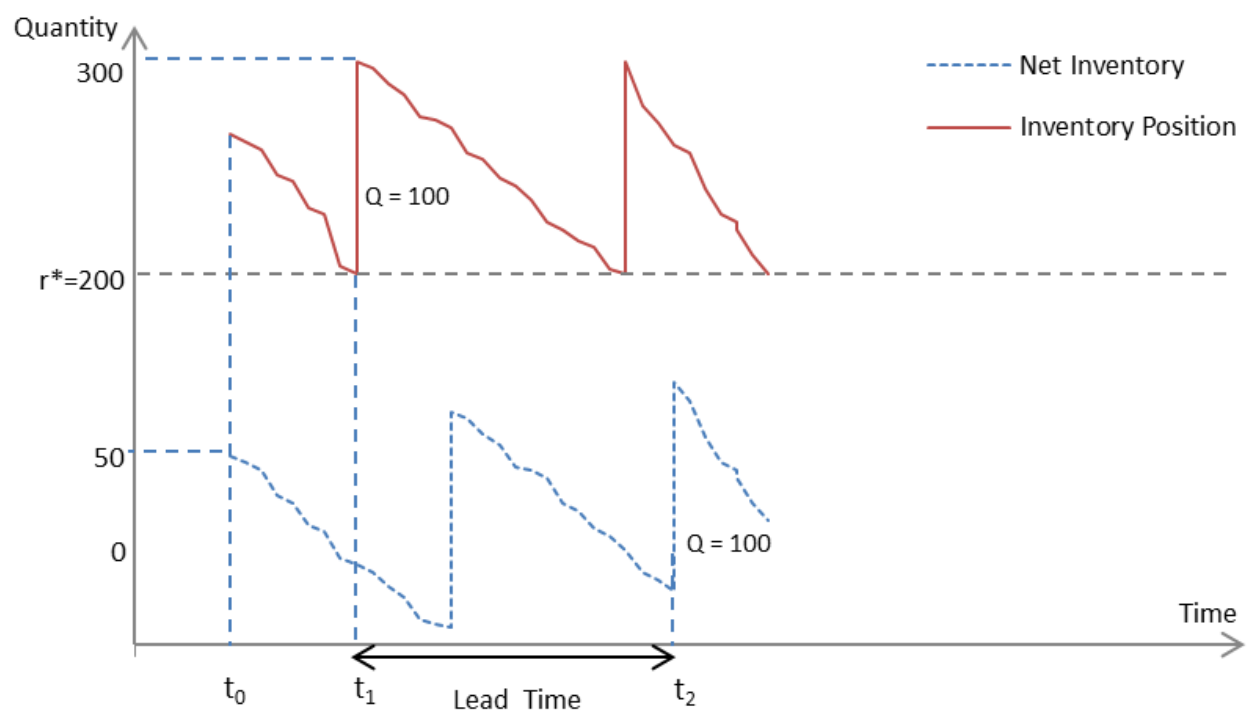

Figure 1. A hypothetical example of Inventory Position vs. Net Inventory Your observation starts at $t_{0} ; t_{1} \& t_{2}$ define the lead time duration

This model was based on students' frequent questions on the reorder point vs. the order quantity such as "If the optimal order quantity is smaller than the reorder point, how can we ever reach the reorder point by placing an order?" Such questions reveal the incomplete understanding of the relationship between the reorder point and order quantity because the reorder point is an abstract concept. That is, reorder point is measured in abstract Inventory Position, and Inventory Position in turn is equal to [On-Hand Inventory - Backorder + On-Order Quantity] and is never a point in time. The order quantity, on the other hand, is conceptually closer to Net Inventory Position. Net Inventory Position exactly reflects the level of On-Hand Inventory when there is no backorder (i.e., in this case, it does have an exact physical representation as it represents what is physically available on the storage shelf). Figure 1 may help students understand that order quantity can be smaller than the reorder point and that can be optimal for the inventory system in the test.

In the second problem (No. 2a, 2b, and 2c) both Group A and Group B students were asked to compute the amounts of expected surplus and shortage for a day and the corresponding expected costs for a day. They were also asked if both the expected surplus cost for a day and 
the expected shortage cost for a day could be positive, and explain the reason. Group A was given the visual model shown in Figure 2.

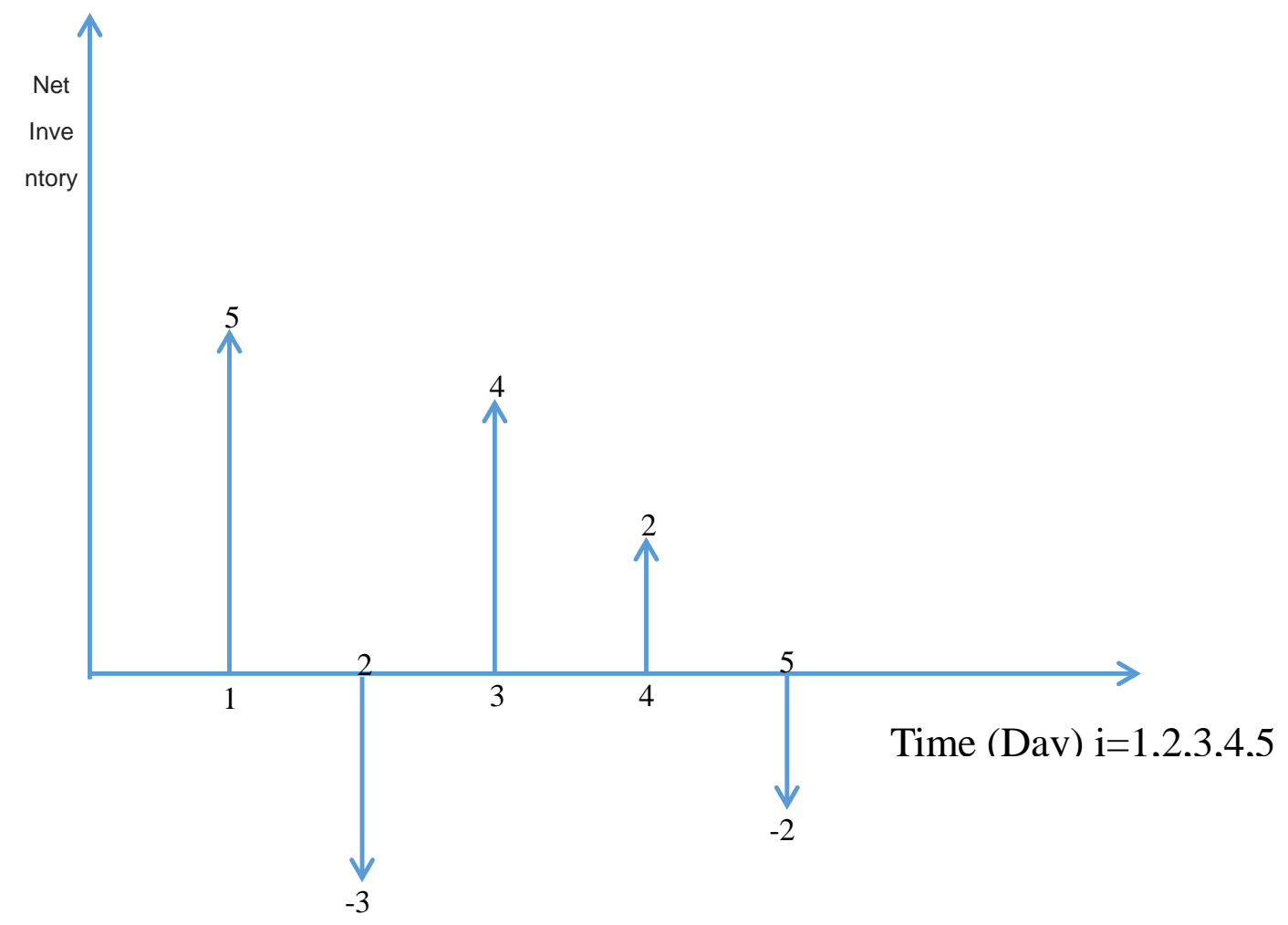

Figure 2. A Representative Realization of Net Inventory over 5 Days

This model is based on students' frequent questions on the average shortage and the average surplus in the context of Net Inventory such as "How can both the average shortage and the average surplus be positive?" At a single point in time, Net Inventory can be positive, zero, or negative, and the corresponding shortage and surplus are never both positive. Average shortage and average surplus must be positive as they are averaged over time (except when the probability of shortage or surplus is artificially and unrealistically set to zero a priori). Hence, such questions reveal the confusion over shortage/surplus at a single point in time and 
the average shortage/surplus. Figure 2 may help students understand that, at a time point, the shortage and surplus cannot both happen while, if an average is taken over time, both must be positive.

The questions for both pre- and post- surveys were exactly the same, and were concerned with students' ability regarding some of the key issues in inventory control. The instructions for the students and the survey are shown below. Student survey responses were based on a Likert scale.

Evaluate your $\underline{\text { ability }}$ to perform each of the following tasks on a numerical scale of 1 to 5 (1 being not at all yet; 5 being fully able as of now).

1. Describe in words the relationship between the inventory position and the net inventory.

$$
12345 \mathrm{NA}
$$

2. Plot the relationship between the inventory position and the net inventory.

$$
12345 \mathrm{NA}
$$

3. Describe the difference between the average levels of shortage and surplus vs. the individual realizations of shortage and surplus.

The pre- survey was conducted one lecture prior to the test while the post-survey was conducted one lecture after the test. Both surveys were voluntary and anonymous except for the check mark indicating group identification.

\section{Results}

The relevant test results for Group A for No.1b, No. 2a, No. 2b, and No. 2c are summarized in Table 1 below. 


\begin{tabular}{|l|c|c|}
\hline Group A Test Results & Average & Standard Deviation \\
\hline No.1b & $5.66 / 10$ & 1.46 \\
\hline No. 2a & $16.50 / 20$ & 2.17 \\
\hline No. 2b & $16.16 / 20$ & 2.50 \\
\hline No. 2c & $6.73 / 10$ & 3.03 \\
\hline
\end{tabular}

Table 1. The Average Scores and Standard Deviations of Group A

The relevant test results for Group B for No.1b, No. 2a, No. 2b, and No. 2c are summarized in Table 2 below.

\begin{tabular}{|l|c|c|}
\hline Group B Test Results & Average & Standard Deviation \\
\hline No.1b & $4.57 / 10$ & 1.33 \\
\hline No. 2a & $16.43 / 20$ & 2.29 \\
\hline No. 2b & $16.14 / 20$ & 2.83 \\
\hline No. 2c & $6.57 / 10$ & 2.59 \\
\hline
\end{tabular}

Table 2. The Average Scores and Standard Deviations of Group B

Given the 44 data points of Group A and the 42 data points of Group B, a two-sample t-test for equal means is justified. That is, the null hypothesis is that the means of both groups are the same while the alternative hypothesis is that the means of both groups are not the same. We note that, for just two groups, a One-Factor ANOVA will lead to the same result as the t-test while, for three or more groups, t-test is not recommended due to an increased chance of committing a type I error.

For No. 1b, the resulting t statistic and the threshold value at $95 \%$ were given by 3.61 and 2.02 , respectively. Hence, we reject the null hypothesis that the means are the same. For No. 2a, No. 2b, and No. 2c, the resulting t statistic and the threshold value were such that we fail to reject the null hypothesis that the means are the same. 
From the t-test result on No. 1b, it appears that Figure 1 was helpful for the students to explain the reason behind their answers. On the other hand, from the t-test results on No. 2a, No. $2 b$, and No. 2 c, it appears that Figure 2 was not helpful. We note that a possible reason is that Figure 2 may lead to misapplications. e.g., add up all the shortages and surpluses and divide by the total number of days. This is similar to "On odd-days, shortages, on even-days, surpluses, hence neither surplus nor shortage on average."

\section{Self-Efficacy Survey Results}

For Group A, 28 and 33 students participated in pre- and post-surveys, respectively while, for Group B, 29 and 34 students participated in pre- and post-surveys, respectively. As both surveys were voluntary and anonymous, responses could not be tracked to an individual. For example, an individual might have participated in the post-survey only (and not pre-survey). Therefore, traditional pre/post assessment tools such as a paired t-test were not applicable. Statistical assessment, however, is still possible in a following way. We demonstrate this by the following example on pre/post-survey Question 1.

The relevant survey results for Group A for Question 1 are summarized in Table 3 below.

\begin{tabular}{|c|c|c|}
\hline Group A Survey Question 1 & Average & Standard Deviation \\
\hline Pre- & $2.786 / 5$ & 0.787 \\
\hline Post- & $3.273 / 5$ & 1.098 \\
\hline
\end{tabular}

Table 3. The Pre- and Post- Survey Results of Group A

The relevant survey results for Group B for Question 1 are summarized in Table 4 below.

\begin{tabular}{|c|c|c|}
\hline Group B Survey Question 1 & Average & Standard Deviation \\
\hline Pre- & $2.724 / 5$ & 1.192 \\
\hline Post- & $2.618 / 5$ & 1.181 \\
\hline
\end{tabular}

Table 4. The Pre- and Post- Survey Results of Group B

Given that the minimum number of the data points is 28 and the maximum number of the data points is 34 , a two-sample t-test for equal means is reasonable. 
First, for the means of pre- Group A and Group B, the null hypothesis is that the means of both groups are the same while the alternative hypothesis is that the means of both groups are not the same. The resulting t statistic and the threshold value at $95 \%$ were given by 0.223 and 2.00 , respectively. Hence, we fail to reject the null hypothesis that the means are the same.

Next, for the means of post- Group A and Group B, the null hypothesis is that the means of both groups are the same while the alternative hypothesis is that the means of both groups are not the same. The resulting t statistic and the threshold value at $95 \%$ were given by 2.06 and 1.998 , respectively. Hence, we reject the null hypothesis that the means are the same.

The fact, that we fail to reject the null hypothesis for the pre-test survey Question 1 while we reject the null hypothesis for the post-test survey Question 1, does indicate that self-efficacy increased due to the inclusion of Figure 1 in the test.

There are numerous explorations possible for the near future such as an exploration for an alternative statistical test that is more straightforward. In addition, further explorations remain regarding Questions 2 and 3, over the significance levels, and with different alternative hypotheses (e.g., upper-tailed instead of two-tailed).

\section{Concluding Remarks and Future Works}

In this paper, we constructed and analyzed an evidence-based practice case to see if visual models led to better understanding of the concepts by students and enhanced their self-efficacy when problems contained abstract concepts without direct physical representations. In the context of inventory control theory, we used a randomized-controlled design research framework implementing the visual models in a quiz. Pre- and post-surveys on student self-efficacy were used to assess the effects of the visual models. Students' performance and self-efficacy surveys were compared between a control group that did not use the visual models and the group with the visual models. The results showed that the visual models did help students understand abstract concepts and improve their self-efficacy. 
This study can serve as a basis for further studies on the extent of visual models helping students develop a complete mental model and on whether better mental models actually lead to better understanding of the domain knowledge and enhance students' self-efficacy. Furthermore, such investigation can be extended to the case of visual feedback (cf. teaching materials; see e.g., Stieff et al. ${ }^{16}$ ).

In addition, this study shows how visual models can be integrated into a course. How these visual models are effectively and efficiently integrated into courses and curricula is another important research issue.

We also note that although this study focused on abstract concepts in industrial engineering, systems engineering, and engineering management, the research findings can be extended to other related areas of engineering, other STEM's, business, management, and economics.

\section{Acknowledgment}

We would like to thank the three anonymous reviewers for constructive and developmental feedback. We also would like to thank former Teaching Assistants, Wenbo Shi, Anuj Mittal, and Anirudh Ramakrishna, of IE 341 Production Systems for their assistance in implementing this project. Finally we would like to thank the Department of Industrial and Manufacturing Systems Engineering for generous support in the form of teaching assistants.

\section{References}

1. Hong, E., O'Neil, H. (1992), Instructional strategies to help learners build relevant mental models in inferential statistics. Journal of Educational Psychology, 84, 150-159.

2. Wheat, I. D. (2007), The feedback method of teaching macroeconomics: is it effective? System Dynamics Review, 23, 391-413.

3. Felder, R. (2002), Learning and teaching styles in engineering education, Engineering Education, 78, 674-681, 1988, Author's Preface.

4. Tall, D. (1991), Intuition and rigor: the role of visualization in the calculus. In Zimmerman 
\& Cunningham (Eds.), Visualization in Mathematics, M.A.A., Notes No. 19, 105-119.

5. Heath, M., Malony, A., Rover, D. (1995), The visual display of parallel performance data, Computer, 28, 21-28.

6. Wood, S. (1996), A new approach to interactive tutorial software for engineering education, IEEE Transactions on Education, 39, 399-408.

7. Novick, L. R., Hurley, S. M., Francis, M. (1999), Evidence for abstract, schematic knowledge of three spatial diagram representations, Memory \& Cognition, 27, 288-308.

8. Haugwitz, M., Nesbit, J. C., Sandmann, A. (2010), Cognitive ability and the instructional efficacy of collaborative concept mapping, Learning and Individual Differences, 20, $536-543$.

9. Baddeley, A. (2012), Working Memory: Theories, Models, and Controversies, Annual Review of Psychology, 63, 1-29.

10. Bull, R., Scerif, G. (2001), Executive functioning as a predictor of children's mathematics ability: Inhibition, switching, and working memory, Developmental Neuropsychology, 19, 273-293.

11. Winn, W. (1989), The design and use of instructional graphics. In H. Mandl \& J. R. Levin (Eds.), Knowledge acquisition from text and pictures (pp. 125-144), Amsterdam, Elsevier. 12. Larkin, J. H., Simon, H. A. (1987), Why a Diagram is (Sometimes) Worth Ten Thousand Words, Cognitive Science, 11, 65-100.

13. Crapo, A., Waisel, L., Wallace, W., Willemain, T. (2000), Visualization and the process of modeling: a cognitive-theoretic view, Proceedings of the sixth ACM SIGKDD international conference on Knowledge discovery and data mining. 218-226.

14. Barwise, J., Etchemendy, J. (1991), Visual information and valid reasoning. In W. Zimmerman \& S. Cunningham (Eds.), Visualization in teaching and learning mathematics (pp. 9-24). Washington, DC: Mathematical Association of America.

15. Pintrich, P., de Groot, E. (1990), Motivational and self-regulated learning components of classroom academic performance, Journal of Educational Psychology, 82, 33-40.

16. Stieff, M., R. Bateman, Jr., and D. Uttal (2007), Teaching and learning with three-dimensional representations, In J. Gilbert (Ed.), Visualization in science education (models and modeling in science education) (pp. 93-120), New York, Springer. 